Urologe $2021 \cdot 60: 1013-1018$

https://doi.org/10.1007/s00120-021-01560-2

Angenommen: 12. Mai 2021

Online publiziert: 17. Juni 2021

๑) Springer Medizin Verlag GmbH, ein Teil von Springer Nature 2021

\title{
Verschärfung der Arbeitsbedingungen durch Kostendruck der Krankenhäuser
}

\author{
Verpflichtende Personalschlüssel für Ärzte?
}

\author{
L. Bellut ${ }^{1} \cdot$ M. Faßbach ${ }^{2} \cdot$ A. Schroeder ${ }^{3} \cdot$ J. Westphal $^{4} \cdot$ A. Beck Be $^{5}$ \\ 'Universitätsklinikum Erlangen, Klinik für Urologie und Kinderurologie, Erlangen, Deutschland \\ ${ }^{2}$ Klinik für Urologie, Urologische Onkologie und Kinderurologie, Helios Klinikum Duisburg, Duisburg, \\ Deutschland \\ ${ }^{3}$ Berufsverband der Deutschen Urologen e. V. (BvDU), Berlin, Deutschland \\ ${ }^{4}$ Klinik für Urologie, Kinderurologie und Urogynäkologie, Krankenhaus Maria-Hilf, Akademisches \\ Lehrkrankenhaus der Heinrich-Heine-Universität Düsseldorf, Alexianer Krefeld GmbH, Krefeld, \\ Deutschland \\ ${ }^{5}$ Klinik für Urologie, Kath. Marienkrankenhaus Hamburg, Hamburg, Deutschland
}

Zusammenfassung

\section{In diesem Beitrag}

- Status quo

- Personaluntergrenzen in der Pflege und deren Folge

- Ampelschema Bundesärztekammer

- Instrumente zur Erfassung des Personalbedarfs

Beispiel Intensivmedizin: Personalplanung muss in ärztlicher Hand bleiben

- Chancen für die Weiterbildung

- Attraktivität der Klinik steigern durch konsequente Einhaltung von Personalvorgaben

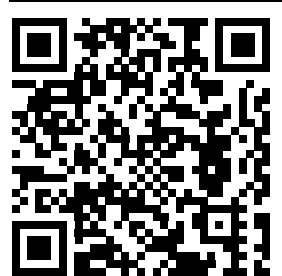

QR-Code scannen \& Beitrag online lesen

Hintergrund: In der aktuellen stationären urologischen Versorgung sind ökonomischer Druck sowie dessen Einfluss auf die urologische Arbeits- und Weiterbildungsbedingungen ein Hauptkritikpunkt junger Urolog*innen. Vor dem Hintergrund eines wachsenden Nachwuchsbedarfs in unserer Fachdisziplin zeichnet sich somit ein dringender Handlungsbedarf ab. Mit der Einführung der Pflegepersonaluntergrenzen droht nun der Abbau von ärztlichem Personal mit dem Ziel einer betriebswirtschaftlich bedingten Kostenreduktion.

Ziel der Arbeit: Ein verpflichtender Personalschlüssel wird häufig genannt um dem Personalabbau in deutschen Klinken entgegenzuwirken. Welche Rolle die Personaluntergrenze hierbei spielt wird im folgenden Artikel erläutert.

Material und Methoden: Wir analysierten die aktuellen Gesetzesentwürfe der Bundesregierung, sowie Positionspapiere und Stellungnahmen bundespolitischer Vertreter wie dem Marburger Bund, Bundesverband Deutscher Urologen und der Bundesärztekammer seit dem Jahr 2018.

Ergebnis und Diskussion: Die Analyse der aktuellen Entwicklungen in der Pflegepolitik zeigt auf, dass als erster Schritt eine bedarfsorientierte Personalbemessung und anschließende Finanzierung im stationären Rahmen unerlässlich ist. Mit der adäquaten Personalbemessung wie im Ampelschema Bundesärztekammer würden sich nicht nur Kliniken als attraktive Arbeitgeber und Weiterbildungsstätten für große Teile der urologischen Ärzteschaft positionieren, sondern auch maßgeblich eine Verbesserung von Arbeitsbedingungen, Patientenversorgung und Patientensicherheit erwirken. Ärztliche Weiterbildung muss weiterhin essenzieller Teil des Klinikalltags bleiben.

\section{Schlüsselwörter}

Weiterbildung · „Diagnosis related groups“ · Assistenzarzt · Work Life Balance · Burn-out

Das Thema Verschärfung der Arbeitsbedingungen trifft alle im Beruf tätigen Urologinnen und hat im Rahmen der Coronapandemie nicht nur das klinische Arbeiten von Ärzt*innen, sondern auch die Patientenversorgung in der Klinik negativ beeinflusst [7]. Zahlreiche Schlagwörter wie Arbeitsverdichtung, Zeit- und Kostendruck,
Personalmangel und verpflichtende Personalschlüssel, Ökonomisierung und Dokumentationspflicht stehen seit Jahren im Flutlicht der Berufspolitik [13]. Mit der Einführung der Pflegepersonaluntergrenzen 2019 und dem Positionspapier der Bundesärztekammer (BÄK) im Sommer 2019 wurde das Thema des Pflegenotstands und 


\section{Infobox}

3. Aachener Internistische Intensivtage 2020: Pflegepersonaluntergrenzen - Sinn oder Unsinn? Referent: Thomas van den Hooven, Münster [28].

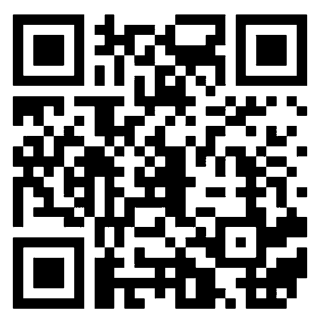

Arbeitsverdichtung breit in den Medien aufgegriffen. Doch bei den ärztlichen Kollegen zeichnet sich ähnliches ab [1]. „Viele Ärztinnen und Ärzte im Krankenhaus arbeiten am Rande der Belastungsgrenzen, was zu einer Gefährdung der Arztgesundheit und der Patientensicherheit führen kann" [19].

\section{॥V Viele Ärzt*innen im Krankenhaus arbeiten am Rande der Belastungsgrenzen}

Der Anteil der Ärzt*innen ist im letzten Jahr gestiegen; jedoch ist die ärztliche Nachwuchsgewinnung im letzten Jahr durch die Coronapandemie ausgebremst worden [8, 9].Zwar stieg die Anzahl der Ärzte seit 2008 um $28 \%$, während die Anzahl an Pflegekräften nur um $10 \%$ bis 2018 wuchs - dies steht jedoch nicht im Verhältnis zur steigenden ärztlichen Arbeitsbelastung und dem zunehmendem Trend an Teilzeitarbeit [20]. Mit der Umstellung auf das DRGSystem ("diagnosis related groups") seit Anfang der 2000er-Jahre, den gestiegenen Anforderungen an Qualität im Rahmen der Zentrenbildung und Zertifizierungen und der deutlichen Verkürzung der Liegezeit ist der Dokumentationsaufwand gestiegen. Jüngst machte der Personalabbau in Helios-Kliniken Schlagzeile, bei

\section{Abkürzungen}

\begin{tabular}{ll} 
BÄK & Bundesärztekammer \\
DRG & "Diagnosis related groups" \\
GeSRU & $\begin{array}{l}\text { German Society of Residents in } \\
\text { Urology e.V. }\end{array}$ \\
PpSG & $\begin{array}{l}\text { Pflegepersonalstärkungsgesetz } \\
\text { PpUGV }\end{array}$ \\
$\begin{array}{l}\text { Pflegepersonaluntergrenzenverord- } \\
\text { nung }\end{array}$ \\
\hline
\end{tabular}

dem Arbeitsverträge nicht verlängert wurden und Stellen nicht oder verspätet nachbesetzt wurden, während gleichzeitig der Vorstand und Aufsichtsrat der FreseniusHauptversammlung für Mai die Erhöhung der Dividendenausschüttung um $5 \%$ auf 88 Cent pro Aktie empfehlen [7, 11].

Dr. Susanna Johna, 1. Vorsitzende des Marburger Bundes, sieht die Einsparung bei Personalkosten nun mehr bei der Ärzteschaft, seitdem die Krankenhäuser diese bedingt durch das Pflegepersonalstärkungsgesetz nicht mehr im pflegerischen Bereich einsparen können. Sie schließt ihren Appell mit: „Wer jetzt ärztliches Personal abbaut, zeigt unmissverständlich, dass das Wohl der Patienten nur im jeweiligen Leitbild im Mittelpunkt steht, nicht aber im konkreten Handeln“ [7]. Den aktuellen Stand bei urologischen Ärzt*innen möchten wir in den folgenden Abschnitten verdeutlichen.

\section{Status quo}

Die Anzahl der Arbeitsstunden im Krankenhaus für das ärztliche Personal ist hoch und überschreitet teilweise die Grenzen des Arbeitszeitgesetzes. Dies zeigt der Marburger Bund-Monitor 2017 anhand einer Befragung von 6172 Ärzt*innen aus dem Krankenhausbereich. Bei $40 \%$ der Befragten lag eine Wochenarbeitszeit von 49-59h, bei $20 \%$ sogar von $60-79 \mathrm{~h}$ vor. Dabei gaben $66 \%$ der Befragten an, nicht ausreichend Zeit für ihre Patienten zur Verfügung zu haben [19].

Dies spiegeln auch aktuelle Umfragen der GeSRU (German Society of Residents in Urology e. V.) in der urologischen Assistenzärzteschaft aus 2020 (in press dieser Ausgabe) und 2015 [5] wider. Hier geben $43 \%$ (vs. $43 \%$ ) eine Wochenarbeitszeit von $50-59 \mathrm{~h}$ an, $25 \%$ (vs. $26 \%$ ) sogar eine Wochenarbeitszeit von $>60 \mathrm{~h}$ an. Zudem berichten $85 \%$ der Befragten über einen Einfluss ökonomischer Erwägungen auf ihre ärztlichen Entscheidungen, was von $45 \%$ als belastend empfunden wird. Die psychosoziale Belastung am Arbeitsplatz wurde mittels des international validierten Modells der beruflichen Gratifikationskrise nach Siegrist erfasst und unter der urologischen Assistenz Ärzteschaft mit einerER-Ratio von 1,79 (2015:1,59) angeben. Dies ist weit über dem Durchschnitt an- derer Berufsgruppen und Disziplinen [16] und stellt ein erhöhtes Risiko beruflich bedingte Erkrankungen, beispielsweise Burnout [6], zu erleiden dar.

Wie deutlich die sich ergebenden gesundheitlichen Beeinträchtigungen sind, spiegelt auch eine Umfrage des Hartmann-Bundes 2018/2019 wieder, in der 1437 Ärzt*innen in der Weiterbildung zu ihrer Arbeitssituation befragt wurden. Fast die Hälfte der Befragten gab an, dass die Personaldecke zwar für den Normalbetrieb ausreichend sei, es jedoch bei Personalausfällen zu großen Problemen käme. Insgesamt 67,6\% der Befragten empfinden ihre Arbeitsbelastung als so groß, dass sie sich negativ auf ihr Privatleben auswirkt, 21,9\% der Befragten befürchten gesundheitliche Beeinträchtigungen und $10,9 \%$ benennen aktuelle gesundheitliche Beeinträchtigungen durch die Arbeitsbelastung. Alarmierend ist auch, dass lediglich ein Viertel der Befragten angab, sich zumindest meistens zufriedenstellend viel Zeit für ihre Patienten nehmen zu können.

\section{Personaluntergrenzen in der Pflege und deren Folge}

Erste Veränderungen in der stationären Krankenhausversorgung ergaben sich jüngst in der Pflege: Strukturelle Untergrenzen in der Krankenpflege sollten laut Bundesgesundheitsministerium 2018 dazu führen, dass Krankenhäuser diesen Umstand ändern müssen - oder aus der Versorgung austreten. Ein Entwurf der Bundesregierung nannte dies einen Entwurf zur Stärkung des Pflegepersonals [10]. Definiert wurde die strukturelle Untergrenze für pflegesensitive Bereiche und ist nun seit dem 01.01.2019 als Pflegepersonaluntergrenzenverordnung (PpUGV) in Kraft getreten [14].

\section{॥) Durch die PpUGV wurden Personaluntergrenzen festgelegt}

Seit 01.01.2019 wurden Personaluntergrenzen durch die Verordnung im Rahmen des Pflegepersonalstärkungsgesetzes (PpSG; $[5,14]$ ) zunächst in den als pflegesensitiv bezeichneten Bereichen Intensivmedizin, Kardiologie, Geriatrie und Unfallchirurgie und seit 01.Februar 2020 
Tab. 1 Grundlegende Prinzipien und Kriterien von Personalvorgaben für Ärzt*innen im Krankenhaus. (Quelle: Positionspapier der BÄK[19])

1. In allen Krankenhäusern müssen verbindliche Personalvorgaben für Ärzt ${ }^{*}$ innen festgelegt werden

2. Personalvorgaben müssen in allen Krankenhausbereichen gelten

3. Maßstab für die Festlegung von Personalvorgaben muss eine Patienten und aufgabengerechte Personalausstattung sein

4. Die Bestimmung der patienten- und aufgabengerechten Personalausstattung erfordert den Einsatz eines standardisierten Bemessungsinstrumentes zur Erfassung des Personalbedarfs

5. Personalvorgaben müssen letztlich für alle in der stationären Patientenversorgung tätigen Berufsgruppen eingeführt werden

6. Die Nicht-Einhaltung von Arbeitszeitgesetzen und Tarifverträgen muss systematisch erfasst und sanktioniert werden

7. Dem vermehrten Bedarf an Ärzt*innen muss Rechnung getragen werden

per Ersatzvornahme mit der PpUGV auch in Innerer Medizin und Kardiologie, allgemeiner Chirurgie und Unfallchirurgie, Herzchirurgie, Neurologie, neurologischer Frührehabilitation, neurologischer Schlaganfalleinheit, pädiatrischer Intensivmedizin und Pädiatrie festgelegt [14]. Hierbei wurden ab 2019 zunächst in den oben genannten Bereichen Pflegepersonaluntergrenzen eingeführt und Sanktionen für das Nichteinhalten dieser Grenzen festgeschrieben. Ab 2020 wurden zudem die Pflegepersonalkosten aus den
DRG-Fallpauschalen ausgegliedert und als zweckgebundenes Pflegebudget im Krankenhausfinanzierungsgesetz verankert. Zur Vermeidung von krankenhausinternen Verschiebungen der Pflegefachkräfte findet zudem eine "Ganzhausbetrachtung" der Pflegepersonalkosten statt. Die zunächst festgelegten Pflegepersonaluntergrenzen sollen mittelfristig von pflegewissenschaftlich fundierten Pflegepersonalbemessungsinstrumenten, welche die Partner der Selbstverwaltung dem Gesetzgeber verbindlich vorschlagen sollen, abgelöst werden [9, 12].

Ein Ziel dieser Maßnahmen ist ein Qualitätserhalt durch Vermeidung weiterer Reduktion der Nurse-to-patient-Relation und hiermit, nach internationaler Datenlage eine sichere und bessere Patientenversorgung [17]. Ein Hauptkritikpunkt, nicht nur aus ärztlicher Sicht bleibt, neben der Honorierung einer Pflegebesetzung auf einem (aktuell unzureichend evaluierten) Minimalmaß, der kurzfristige Anreiz zur Einsparung von Lohnkosten bei nicht-ärztlichem und ärztlichem übrigen Krankenhauspersonal.[2]. Diskutiert wurde dies ebenfalls auf den 3. Aachener Internistischen Intensivtagen 2020 mit eben dieser Forderung (- Infobox 1).

\section{Ampelschema Bundes- ärztekammer}

In dem Positionspapier der BÄK heißt es: „Die Bundesärztekammer sieht sich daher in der Pflicht, Ärztinnen und Ärzte sowie Patientinnen und Patienten vor den ne- gativen Auswirkungen einer unzureichenden Personalpolitik zu schützen“" [19]. Aus diesem Grund wurden sieben Forderungen für grundlegende Prinzipien und Kriterien von Personalvorgaben aufgestellt (- Tab. 1). Parallel dazu wurde ein Ampelschema entwickelt, welches die Begrifflichkeiten Mindestpersonalvorgaben, Personaluntergrenzen, adäquate, ausreichende oder notwendige Personalausstattung definiert, wertet und hierarchisch bewertet. Besteht eine optimale patienten- und aufgabengerechte ärztliche Personalausstattung, ist genügend Zeit für die ärztliche Versorgung vorhanden; je weniger ärztliches Personal vorhanden ist, je weiter die Anzahl von einer adäquaten auf eine Mindestanzahl von Ärzt*innen sinkt, desto weniger Zeit ist vorhanden.

\section{》) Personaluntergrenzen dürfen nicht mit Mindestpersonalgrenzen gleichgesetzt werden}

Wird die Personaluntergrenze unterschritten, ist die Personalausstattung inakzeptabel, ist keine Zeit mehr für ärztliche Versorgung vorhanden. Bei diesem Ist-Zustand der fehlenden ärztlichen Versorgung für die Gesamtheit der Patient*innen ist die Arbeitsbelastung der einzelnen Ärzt ${ }^{*}$ innen maximal; sie nimmt mit zunehmender Personaldecke hin bis zur optimalen patienten- und aufgabengerechten ärztlichen Personalausstattung gegenläufig wieder ab (-Tab. 2). Personaluntergrenzen dürfen nicht aufgrund von ökonomischem Druck mit Mindestpersonalgrenzen gleich-

Hier steht eine Anzeige. 
Tab. 2 Definition der Begrifflichkeiten im Ampelschema der Bundesärztekammer (BÄK) zu Personalvorgaben im Krankenhaus [19]

\begin{tabular}{l|l}
\hline Personaluntergrenze & $\begin{array}{l}\text { "Die Personaluntergrenze bezeichnet einen Grenzwert. Ein Unterschreiten der Personaluntergrenze gefährdet die Patien- } \\
\text { tensicherheit und bedeutet für die Ärzt*innen eine unzumutbare Arbeitssituation und Arbeitsbelastung. Eine Personalbe- } \\
\text { setzung unterhalb der Personaluntergrenze muss sofortige Gegenmaßnahmen nach sich ziehen" }\end{array}$ \\
$\begin{array}{l}\text { Mindestpersonal- } \\
\text { vorgaben }\end{array}$ & "Die Mindestpersonalvorgaben werden durch Arbeitszeitgesetz und Tarifverträge vorgegeben" \\
\hline $\begin{array}{l}\text { Adäquate Personalaus- } \\
\text { stattung }\end{array}$ & $\begin{array}{l}\text { "Die ärztliche Personalausstattung, mit der nötige direkte/patientenseitige ärztliche Aufgaben abgedeckt sind. Für Aufga- } \\
\text { ben der indirekten Patientenversorgung sowie über die direkte Patientenversorgung hinausgehende Aufgaben steht nicht } \\
\text { ausreichend Personal zur Verfügung. Für die Patienten kann es während des Krankenhausaufenthalts zu Einschränkungen } \\
\text { in der Versorgung und zu unnötigen Wartezeiten kommen. Die Arbeitsbelastung der Ärzt*innen sind hoch“ }\end{array}$ \\
$\begin{array}{l}\text { Optimale patienten- } \\
\text { und aufgabengerechte } \\
\text { ärztliche Personalaus- } \\
\text { stattung }\end{array}$ & $\begin{array}{l}\text { "Die ärztliche Personalausstattung, mit der alle direkten und indirekten patientenseitigen ärztlichen Aufgaben sowie auch } \\
\text { die weiteren ärztlichen Aufgaben und Pflichten abgedeckt sind (z. B. Fort- und Weiterbildung, Erfüllung rechtlicher und } \\
\text { gesetzlicher Aufgaben, Verwaltungsaufgaben, Team- und Mitarbeiterentwicklung). Die Patienten erhalten zügig alle not- } \\
\text { wendigen Untersuchungen und Interventionen. Es gibt ausreichend viel Zeit für Fragen und partizipative Gespräche" }\end{array}$
\end{tabular}

gesetzt werden und als der neue Standard gelten.

\section{Instrumente zur Erfassung des Personalbedarfs}

Beispiel Intensivmedizin: Personalplanung muss in ärztlicher Hand bleiben

Zur Bestimmung der Personalausstattung fordert die BÄK den Einsatz eines standardisierten Bemessungsinstruments zur Erfassung des Personalbedarfs [19]. In der Pflege wurde ein vergleichbares Instrument für verschiedenste Akutstationen inklusive Urologie bereits aktualisiert und überprüft [25]. Im Bereich der ärztlichen Personalausstattung ist bisher lediglich ein Tool zur Personalbedarfskalkulation der Intensivstationen entwickelt worden [26]. In der aktualisierten Version von 2018 konnte der komplexen Personalplanung in Zeiten von Mutterschutz, Eltern- und Teilzeit, sowie Überstundenausgleich durch Freizeit Rechnung getragen werden. Auch sozialund arbeitsrechtlichen Rahmenbedingungen wurden berücksichtigt. Der ärztliche Arbeitsaufwand wurde in Basis- und Zusatzaufwand abhängig von der Krankheitsschwere der Patienten unterteilt.

Auch nicht-patientenbezogene Aufgaben wie Administration, Kommissionsarbeit und Aufgaben im Rahmen von Verordnungspflichten fließen in die Berechnung mit ein, wie Weiss et al. [26] beschreiben. Die Autoren kommen zu dem Schluss, dass „mit Hilfe des Personaltools Intensivmedizin der Personalbedarf leistungsorientiert ermittelt werden kann". Dies sollte auch für urologische Kliniken etabliert werden, da so objektiv der Personalbedarf abgebildet werden kann. Nur durch Entwicklung eines solchen Instruments von ärztlicher Seite aus kann der hohe Anteil nicht-patientenbezogener Aufgaben an der ärztlichen Arbeit, eine realistische Beurteilung des ärztlichen Arbeitsaufwands und die Zeit für Weiterbildung berücksichtigt werden. So könnte einer zunehmenden Arbeitsverdichtung entgegengewirkt werden, welche als Mitursache eines vermehrten Burnouts junger Ärzt*innen angesehen wird [21]. Strukturprobleme könnten durch genanntes Tool nicht gelöst werden, allerdings könnte durch ein systematisches und professionelles Personalmanagement der Personalbestand, seine Qualität und die Entwicklungsfähigkeit gesichert werden [26]. Auch die Sicherstellung verlässlicher Zeit zur Weiterbildung erscheint hierdurch denkbar.

\section{Chancen für die Weiterbildung}

Die ärztliche Weiterbildung wird aktuell nur indirekt von den Krankenkassen über das DRG-System finanziert [24]. Tatsächlich kostet diese Zeit im Operationssaal (OP) und ist damit bislang ein Kostenfaktor [23]. Huber et al. [15] zeigten 2020, dass der tatsächliche Anteil durch chirurgische Assistenzärzt*innen durchgeführter Lehreingriffe mit 24,4\% alarmierend gering ausfiel. Als häufigster Grund hierfür wurden in der Studie "organisatorische Gründe" angegeben, wobei sich in 77,9\% dieser Fälle keine Assistenzärzt*in im OP befand. Den Grund für die Abwesenheit der Assistenzärzt*innen nennen die Autoren nicht. Es darf allerdings spekuliert werden, dass zur Aufrechterhaltung der
Patientenversorgung auf der Station, den Ambulanzen oder der Notaufnahme die Assistenzärzt*innen außerhalb des OP eingeteilt waren.

\section{॥ Teilzeitarbeit und die} Vereinbarkeit von Familie und Beruf spielen eine wichtige Rolle

Kommt es durch Kostendruck der Krankenhäuser zu einer Reduzierung des real anwesenden ärztlichen Personals, so schwindet auch die Zeit für Aus- und Weiterbildung. Durch einen verpflichtenden und an die Fülle der Aufgaben angepassten Personalschlüssel ließe sich eine curriculare und zeitgerechte Weiterbildung ermöglichen. Gute strukturierte Weiterbildung sollte hierbei sowohl finanziell als auch durch öffentliche Reputation entlohnt werden [18].

In den nächsten Jahren gilt es gleich mehrfache Herausforderungen zu bewältigen: Mit dem demographischem Wandel in Deutschland wird es mehr ältere Patienten geben, die einer ärztlichen Behandlung bedürfen. Zum anderen sind $47 \%$ alle Ärzt*innen laut der Ärztestatistik über 50 Jahre alt und werden in den nächsten zwei Jahrzehnten in den Ruhestand treten. Die Anzahl der berufstätigen Ärzte ist 2020 zwar um 1,7 \% im Vergleich zum Vorjahr auf 409.000 gewachsen, doch titelt das Deutsche Ärzteblatt nicht umsonst: „Mehr Köpfe, nicht mehr Arztstunden" [4].

Die Statistik zeigt ebenfalls: Teilzeitarbeit und die Vereinbarkeit von Familie und Beruf spielen eine wichtige Rolle. Die Verschärfung der Arbeitsbedingungen konnte die letzte MB-Monitorumfrage auch in der Teilzeitarbeit abbilden: 
durchschnittlich arbeiten diese Mitarbeiter 39,6h/Woche. Nicht miteinbezogen ist hierbei die Arbeitsbelastung durch die COVID-19-Pandemie („,coronavirus disease 2019“, [4]). Umso wichtiger ist es, dass Weiterbildung auch weiter im klinischen Alltag berücksichtigt wird.

\section{Attraktivität der Klinik steigern durch konsequente Einhaltung von Personalvorgaben}

Mit der Einhaltung von Personalvorgaben kann der Krankenhausstandort gestärkt werden. Die Klinik bietet Patientensicherheit, indem Personal Schutz vor Überlastung gewährleistet wird, Überstunden verhindert, Patienten zügig alle notwendigen Untersuchungen und Interventionen erhalten, Aufklärung durch die ärztlichen Behandler erfahren und somit das Vertrauen der Bevölkerung gewonnen wird. Gerade mangelnde Zeit und Aufklärung von Ärzt*innen wird sehr häufig in Patientenbeschwerden angeführt. Ist die Arbeitsüberlastung der Ärzt*innen überschaubar, sind Fehler in der Patientenversorgung reduziert - dies wurde bereits in der Pflege untersucht [1]. Umgekehrt kann Übermüdung des Arztes zu potenziellen Behandlungsfehlern führen [3].

\section{I) Die patienten- und aufgaben- gerechte Personalausstattung führt mittelbar zu ökonomischen Vorteilen}

Die patienten- und aufgabengerechten Personalausstattung führt auch mittelbar zu ökonomischen Vorteilen [19]. Mit dem Erhalt der Arbeitszufriedenheit der Ärzt*innen gewinnt die Klinik an Attraktivität; weniger Personalfluktuation bedeutet, dass Zeit in Patientenversorgung investiert werden kann statt in Einarbeitung oder Fehlermanagement. Gleichzeitig sinken körperliche und emotionale Belastung von jungen Ärzt*innen und die Gefahr eines Burn-outs wird reduziert [13]. Dies findet Anschluss zur Zukunftsoffensive 2025 der Deutschen Gesellschaft für Urologie mit der Erarbeitung alternativer Arbeitszeit- und Dienstmodelle, Förderung der Arbeitszufriedenheit ohne Vernachlässigung der Aus- und Weiterbildung [27]. Ein interessanter Aspekt

\section{Exacerbation of working conditions due to the economic pressures of} hospitals. Mandatory staffing ratios for physicians?

Background: In current hospital-based urological care, economic pressure and its influence on urological working and further training conditions are a main point of criticism among young urologists. Against the background of a growing need for young talent in our specialist discipline, there is an urgent need for action. With the introduction of the lower limits for nursing staff, there is now a threat of a reduction in the number of physicians with the aim of reducing costs for business reasons.

Objective: A mandatory staffing ratio is often mentioned to counteract the downsizing in German clinics. The role of the lower staffing limit is explained in the following article.

Materials and methods: We have analyzed the current draft laws of the federal government, as well as position papers and statements from federal political representatives such as the Marburger Bund, Bundesverband Deutscher Urologen and the German Medical Association (Bundesärztekammer) since 2018.

Results and conclusion: The analysis of current developments in nursing policy shows that the first step is a needs-based assessment of personnel and subsequent financing in the clinic setting. With adequate staffing, as in the traffic light scheme of the German Medical Association, not only would clinics position themselves as attractive employers and training centers for large parts of the urological medical profession, but would also significantly improve working conditions, patient care and patient safety. Medical training must remain an essential part of everyday hospital life.

\section{Keywords}

Specialist training · Diagnosis related groups $\cdot$ Medical residency $\cdot$ Work-life balance $\cdot$ Burnout

in diesem Zusammenhang ist: Eine gute Interprofessionelle Zusammenarbeit zwischen Ärzteschaft und Pflegenden ist ein protektiver Faktor für die Gesundheit dieser im Krankenhaus [22]. Bislang wird diese als gering eingestuft; mit der Verbesserung der Zusammenarbeit könnten jedoch die Arbeitszufriedenheit verbessert, Berufsaufgabe verhindert und Burnout vermindert werden. Welche Rolle der ärztliche Personalschlüssel dabei spielt, gilt es weiterhin zu klären - der Schlüssel für Pflegekräfte steht bereits.

\section{Fazit für die Praxis}

- Auch 2 Jahre nach dem Positionspapier der Bundesärztekammer (BÄK) sind grundlegende Prinzipien und Kriterien für ärztliche Personalvorgaben im Krankenhaus und in der Urologie nicht erfüllt.

- Eine qualitativ hochwertige Versorgung ist nur mit einer patienten- und aufgabengerechten Ausstattung möglich.

- Personaluntergrenzen dürfen nicht mit Mindestpersonalgrenzen gleichgesetzt werden und als der neue Standard gelten.

- Instrumente zur Bemessung des Personalbedarfs müssen in der Urologie für eine bessere Transparenz dringend etabliert werden.
- Zeit für Weiterbildung muss in der Personalplanung mitgedacht und mitgeplant werden.

- Professionelles und systematisches Personalmanagement von ärztlicher Seite ist notwendig, um die Qualität des Personalbestands und seine Entwicklungsfähigkeit zu erhalten.

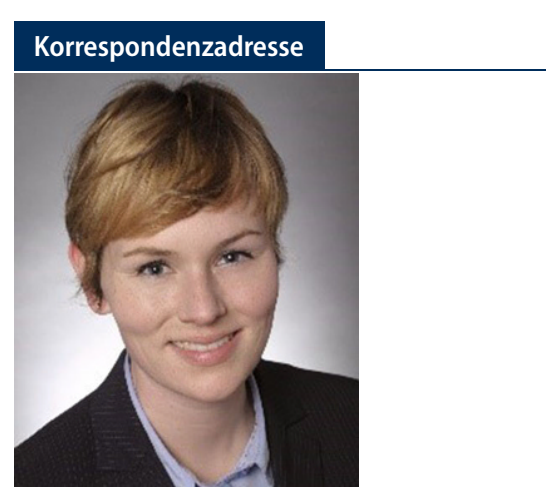

Dr. med. L. Bellut

Universitätsklinikum Erlangen, Klinik für Urologie und Kinderurologie

Krankenhausstraße 12, 91054 Erlangen,

Deutschland

laura.bellut@uk-erlangen.de 


\section{Einhaltung ethischer Richtlinien}

Interessenkonflikt. L. Bellut, M. Faßbach, A. Schroeder, J. Westphal und A. Beck geben an, dass kein Interessenkonflikt besteht.

Für diesen Beitrag wurden von den Autoren keine Studien an Menschen oder Tieren durchgeführt. Für die aufgeführten Studien gelten die jeweils dort angegebenen ethischen Richtlinien.

\section{Literatur}

1. Aiken LH, Sloane DM, Bruyneel L et al (2014) Nurse staffing and education and hospital mortality in nine European countries: a retrospective observational study. Lancet 383:1824-1830

2. Stellungnahme des Marburger Bund Bundesverbandes zu dem Referentenentwurf des Bundesministeriums für Gesundheit Verordnung zur Festlegung von Pflegepersonaluntergrenzen in pflegesensitiven Bereichen in Krankenhäusern für das Jahr 2021 (PflegepersonaluntergrenzenVerordnung - PpUGV) (Stand Referentenentwurf: 1. Okt. 2020).

3. Bernd G (2015) Wenn übermüdeten Ärzten Behandlungsfehler unterlaufen. Dtsch Arztebl 112(26):4-5

4. Blum M (2021) Mehr Köpfe, nicht mehr Arztstunden. Dtsch Arztebl 118:762

5. Borgmann H, Arnold HK, Meyer CP et al (2018) Training, research, and working conditions for urology residents in Germany: a contemporary survey. Eur Urol Focus 4:455-460

6. Buddeberg-Fischer B, Klaghofer R, Stamm M et al (2008) Work stress and reduced health in young physicians: prospective evidence from Swiss residents. Int Arch Occup Environ Health 82:31-38

7. Marburger Bund Zeitung 74(5/16):G7916

8. Bundesärztekammer (2021) Ergebnisse der Ärztestatistik zum 31.12.2020. Corona-Pandemie bremst ärztliche Nachwuchsgewinnung

9. Bundesregierung Deutschland (2020) Konzertierte Aktion Pflege. Erster Bericht zum Stand der Umsetzung der Vereinbarungen der Arbeitsgruppen 1 bis $5,51-92$

10. Bundesregierung GD (2018) Entwurf eines Gesetzes zur Stärkung des Pflegepersonals (Pflegepersonal-Stärkungsgesetz-PpSG), S1-136

11. https://Geschaeftsbericht.Fresenius.De/2020/anUnsere-Aktionaere/Fresenius-Aktie/.Zugegriffen: 30. Apr. 2021

12. https://www.Aerzteblatt.De/Nachrichten/ 123310/Spahn-Stellt-Roadmap-FuerPflegepersonalbemessung-in-Aussicht. Zugegriffen: 28. Apr. 2021

13. https://www.Aerzteblatt.De/Treffer? Mode $=S \& W o=1041 \&$ Typ $=1 \& N i d=110968 \&$ $\mathrm{S}=$ Kostendruck\&S=Arbeitsbedingungen \& S=Personalschl\%Fcssel.Zugegriffen: 28. Apr. 2021

14. https://www.Bundesgesundheitsministerium. De/Personaluntergrenzen.html. Zugegriffen: 28. Apr. 2021

15. Huber T, Richardsen I, Klinger $C$ et al (2020) See (n)One, Do (n)One, Teach (n)One: Reality of Surgical Resident Training in Germany. World J Surg 44:2501-2510

16. Joachim SC, Bitzinger D, Arnold $\mathrm{H}$ et al (2020) Work and training of young doctors in Germany-a comprehensive analysis of survey results from six disciplines. Gesundheitswesen 82:227-235
17. Justiz BF (2021) Verordnung zur Festlegung von Pflegepersonaluntergrenzen in pflegesensitiven Bereichen in Krankenhäusern für das Jahr 2021

18. Konig J, Aeishen S, Cebulla A et al (2019) Quality instead of quantity improves medical education. Urologe A 58:877-880

19. Krankenhaus BaPFÄl (2019) Prinzipien und Kriterien zu Personalvorgaben für Ärztinnen und Ärzte im Krankenhaus. In: Positionspapier, S5

20. Osterloh F (2019) Krankenhaus: Personalvorgaben für Ärzte. Dtsch Arztebl 116:1810

21. Raspe M, Koch P, Zilezinski M et al (2020) Working conditions and health status of young physicians and nurses in German hospitals. Bundesgesundheitsblatt Gesundheitsforschung Gesundheitsschutz 63:113-121

22. Richter-Kuhlmann E (2019) Arbeitsbedingungen im Krankenhaus. Burn-outschon beim Nachwuchs. Dtsch Arztebl 48:2222-2224

23. Ruhotina N, Dagenais J, Gandaglia G et al (2014) The impact of resident involvement in minimallyinvasive urologic oncology procedures. Can Urol Assoc J 8:334-340

24. Schroder W, Welcker K (2010) Financing and control of surgical training. Chirurg 81:31-37

25. Steffen F (2020) Pre-Test einer modernisierten PflegepersonalRegelung für Erwachsene PPR 2.0. In: Abschlussbericht DKG, S 1-50

26. Weiss MMG, Vagts D, Schleppers A, Leidinger W, Klöss T, Iber T (2018) Personalbedarfs kalkulation Intensivmedizin Überarbeitung der Kalkulationsgrundlagen für den ärztlichen Dienst aus dem Jahr 2012. Anasthesiol Intensivmed 59:458-483

27. Arbeitskreis Angestellte Arzte Im Berufsverband Der Deutschen Urologen Als V, Westphal J, Struck et al (2019) Workload, job satisfaction, Work Time Act-what is urology doing? Urologe A 58:881-884

28. https://www.youtube.com/watch?v=UJtpcisnXw.Zugegriffen:30.Apr. 2021 